\title{
A Multimodal Approach to Evaluate for Cardiac Metastasis in a Case of Non-Small Cell Lung Cancer
}

\author{
Nadeem Bilani ${ }^{a} \quad$ Leah Elson $^{a}$ Felipe Martinez ${ }^{b}$ Diego Sadler ${ }^{c}$ \\ Zeina Nahleh ${ }^{a} \quad E^{2}$ lizabeth Elimimian ${ }^{a} \quad E^{2}$ an Alley ${ }^{a}$ \\ aMaroone Cancer Center, Cleveland Clinic Florida, Weston, FL, USA; bepartment of \\ Radiology, Cleveland Clinic Florida, Weston, FL, USA; CHeart and Vascular Center, \\ Cleveland Clinic Florida, Weston, FL, USA
}

\author{
Keywords \\ Oncology · Cardiac metastasis · Cardiac magnetic resonance imaging
}

\begin{abstract}
Malignancies have demonstrated the ability to metastasize to cardiac tissue. However, an optimal diagnostic algorithm for cardiac tumors has not yet been established, due at least in part to the scarcity of symptomatic cases. Several case reports describe how the usage of ${ }^{18} \mathrm{~F}-\mathrm{la}$ beled fluorodeoxyglucose positron emission tomography ( $\left({ }^{18} \mathrm{~F}-\mathrm{FDG} \mathrm{PET}\right)$ incidentally revealed cardiac neoplasia. This modality, which indicates uptake sites of the radioisotope ${ }^{18} \mathrm{~F}-\mathrm{FDG}$, allows for whole-body imaging and is often used for preoperative determination of malignant metastasis or for assessing response to therapy over time. However, findings of false positivity are often reported due to increased FDG avidity caused by a range of other, nonmetastatic processes, most notably inflammation and infection. In this case report, an 84-year-old male with stage IV non-small cell lung cancer presented a clinical course, echocardiogram, and ${ }^{18} \mathrm{~F}-\mathrm{FDG}$ PET-CT findings that were suggestive of endocardial metastasis. Nine months into therapy, after extensive consultation, the patient finally consented to a more complete workup using cardiac MRI (CMRI), which showed no evidence of cardiac metastasis. This case report supports the utility of $\mathrm{CMRI}$ as a means of further interpreting intracardiac, localized FDG uptake foci in PET-CT findings, in order to avoid false positivity and further refine proposed cardiac differential diagnoses in cancer patients.


Bilani et al.: Multimodal Imaging Approach to Mitigate False Positivity in Cardiac Metastasis

\section{Introduction}

Multiple malignancies have demonstrated the ability to metastasize to cardiac tissue. While this metastatic course is uncommon, the incidence has increased over the last decade due to improved survival rates among cancer patients [1]. In postmortem evaluations, cardiac metastasis has been reported to occur in up to $9.1 \%$ of patients with advanced cancers [2]. The most common primary malignancies associated with cardiac metastasis reported in the literature are lymphomas, melanoma, breast cancer, and lung neoplasms [3-5]. In lung cancer, specifically, cardiac metastasis may occur through lymphatic or hematogenous channels, as well as by direct extension [6].

While the risk of complications from cardiac metastasis is relatively low, the potential clinical manifestations range from mild to life-threatening. When present, common signs and symptoms may include nonspecific symptoms such as dyspnea, palpitations, and atrial arrhythmias. In the extreme, intracavitary heart masses may cause cardiogenic shock due to outflow obstruction, pulmonary emboli (if originating in the right heart), and stroke (if originating in the left heart) [2].

To date, an optimal diagnostic algorithm for cardiac tumors has not yet been established, due at least in part to the scarcity of symptomatic cases [7]. Associated electrocardiogram findings indicating possible cardiac involvement include ST changes, heart blocks, or newonset arrhythmias, though none of these are pathognomonic [8]. Therefore, cardiac imaging provides an increasingly important means of evaluation for progressive malignant processes.

Echocardiography is generally the preferred initial cardiac imaging modality, allowing characterization of the site, size, and shape of cardiac tumors [9]. However, echocardiography is limited by a poor acoustic window in certain patients, no tissue characterization, and operator dependence [10]. Additionally, delineation of the endomyocardial border can be challenging [11], which limits the capacity of this modality to evaluate intracardiac tumors.

A number of case reports describe instances whereby ${ }^{18} \mathrm{~F}$-labeled fluorodeoxyglucose positron emission tomography $\left({ }^{18} \mathrm{~F}-\mathrm{FDG}\right.$ PET) incidentally revealed cardiac neoplasia [1218]. This modality, which highlights uptake sites of the radioisotope ${ }^{18} \mathrm{~F}-\mathrm{FDG}$, allows for whole-body imaging and is often used for preoperative determination of malignant metastasis or for assessing response to therapy over time [9]. However, findings of false positivity are often reported due to increased FDG avidity caused by a range of other, nonmetastatic processes, most notably inflammation and infection [10].

The gold standard for the definitive diagnosis of cardiac tumors is tissue examination following biopsy or specimen collection during surgery. However, this diagnostic power may oftentimes be overshadowed by the risks of invasiveness of biopsy and, to a greater extent, surgery. Cardiac MRI (CMRI), on the other hand, is quickly becoming the reference standard for anatomic and tissue evaluation of cardiac neoplasia [11]. To our knowledge, however, there are no specific guidelines that describe the use of CMRI in the workup for potential cardiac metastasis.

Herein, we present the case of an 84-year-old male with stage IV non-small cell lung cancer (NSCLC) whose clinical course, echocardiogram, and ${ }^{18}$ F-FDG PET-CT findings were suggestive of endocardial metastasis. Nine months into therapy, after extensive consultation, the patient finally consented to a more complete workup using CMRI, which showed no evidence of cardiac metastasis. Prior to the MRI findings, the clinicians had held a high suspicion of progressive cardiac involvement, which would have directed a different management plan for this patient. This case report supports the utility of CMRI as a means of further interpreting intracardiac, localized FDG-uptake foci in PET-CT findings in order to avoid false positivity and further refine proposed cardiac differential diagnoses in cancer patients. 
Bilani et al.: Multimodal Imaging Approach to Mitigate False Positivity in Cardiac Metastasis

\section{Case Presentation}

The presented patient is an 84-year-old male with known hypertension, dyslipidemia, mitral valve prolapse with a focal flail segment (portion of P2), and a smoking history of 40 pack-years (quit 20 years prior to the first encounter). He was emergently admitted after three episodes of exertional dyspnea over a 1-month period had progressed to dyspnea occurring at rest. He was found to have a large left pleural effusion on chest radiography. A CT scan confirmed the effusion to be overlying a $3.5-\mathrm{cm}$ anterior left upper lobe (LUL) consolidation. There was also associated near-complete left lung atelectasis. Thoracentesis of 2,500 $\mathrm{mL}$ of pleural fluid was performed, and a sample sent for microbiologic and cytologic analysis. The fluid was exudative by standard parameters. The cultures were negative for acid-fast bacilli, fungi, and anaerobic organisms; cytology was negative for the presence of malignant cells. The next day, the patient had been stabilized and was comfortable. He was consequently discharged with instructions to return for follow-up with a pulmonologist at the clinic and a prescription of oral azithromycin for possible community-acquired pneumonia.

An ${ }^{18}$ F-FDG PET-CT was performed, identifying an FDG-avid LUL mass. There was also mediastinal and bilateral hilar adenopathy, as well as an intracardiac focus of FDG avidity. This was further described as a 1.5-cm FDG-avid hypodense nodularity within the anterior papillary muscle, with a maximum standard uptake value of 112.3. The patient refused further evaluation with CMRI, as recommended by the medical team due to anxiety surrounding the machine and test. A brain MRI was thus also not performed, but the suspicion for brain metastasis was low due to the absence of neurologic symptoms and an unremarkable neurologic physical examination.

At the time of admission, the patient was also found to be hyponatremic. This was considered a paraneoplastic syndrome of inappropriate ADH secretion, to be managed with treatment of the primary malignancy. Biopsy of the LUL mass showed a poorly differentiated carcinoma with sarcomatoid features, a rare histological subtype of NSCLC known to have a poor prognosis. Molecular profiling demonstrated high PD-L1 expression ( $>50 \%)$, wild-type EGFR, and negative ALK and ROS1 mutations. The patient was consequently started on immunotherapy with $200 \mathrm{mg}$ intravenous (IV) pembrolizumab every 21 days. A second PET-CT was performed 2 weeks after his third cycle of immunotherapy, showing a partial response to treatment as well as resolution of the intracardiac focus. Four months into his treatment, the patient experienced bilateral leg swelling. He was evaluated for thrombosis and cardiogenic causes of peripheral edema. Lower-extremity Doppler ultrasound showed no evidence of deep vein thrombosis. The erythrocyte sedimentation rate $(88 \mathrm{~mm} / \mathrm{h}), \mathrm{C}$-reactive protein level $(2.9 \mathrm{mg} / \mathrm{dL})$, troponin $\mathrm{T}$ level $(<0.010 \mathrm{ng} / \mathrm{mL})$, EKG (unremarkable), and echocardiogram (stable severe mitral regurgitation and a left ventricular ejection fraction $>60 \%$ ) showed no evidence of myocarditis. The peripheral edema was managed with compression stockings and physical therapy. Two subsequent interim PET-CT scans, spaced 2 months apart, also did not reveal intracardiac FDG avidity; however, 9 months into treatment, his PET-CT showed a recurrent focus of FDG avidity in the left ventricle at the same site as initially noted despite continuing response at other metastatic sites (Fig. 1). The patient was reeducated on the importance of anatomic imaging once again with the explanation that confirmed cardiac metastasis may warrant a treatment regimen change, and possibly even a surgical intervention. The patient underwent CMRI with and without IV contrast 1 month later, showing no evidence of an intracardiac mass.

At the time of this report, the patient had received 15 cycles of pembrolizumab, and as a result of the CMRI findings, he did not require any alteration of therapy or surgical intervention. 
Bilani et al.: Multimodal Imaging Approach to Mitigate False Positivity in Cardiac Metastasis

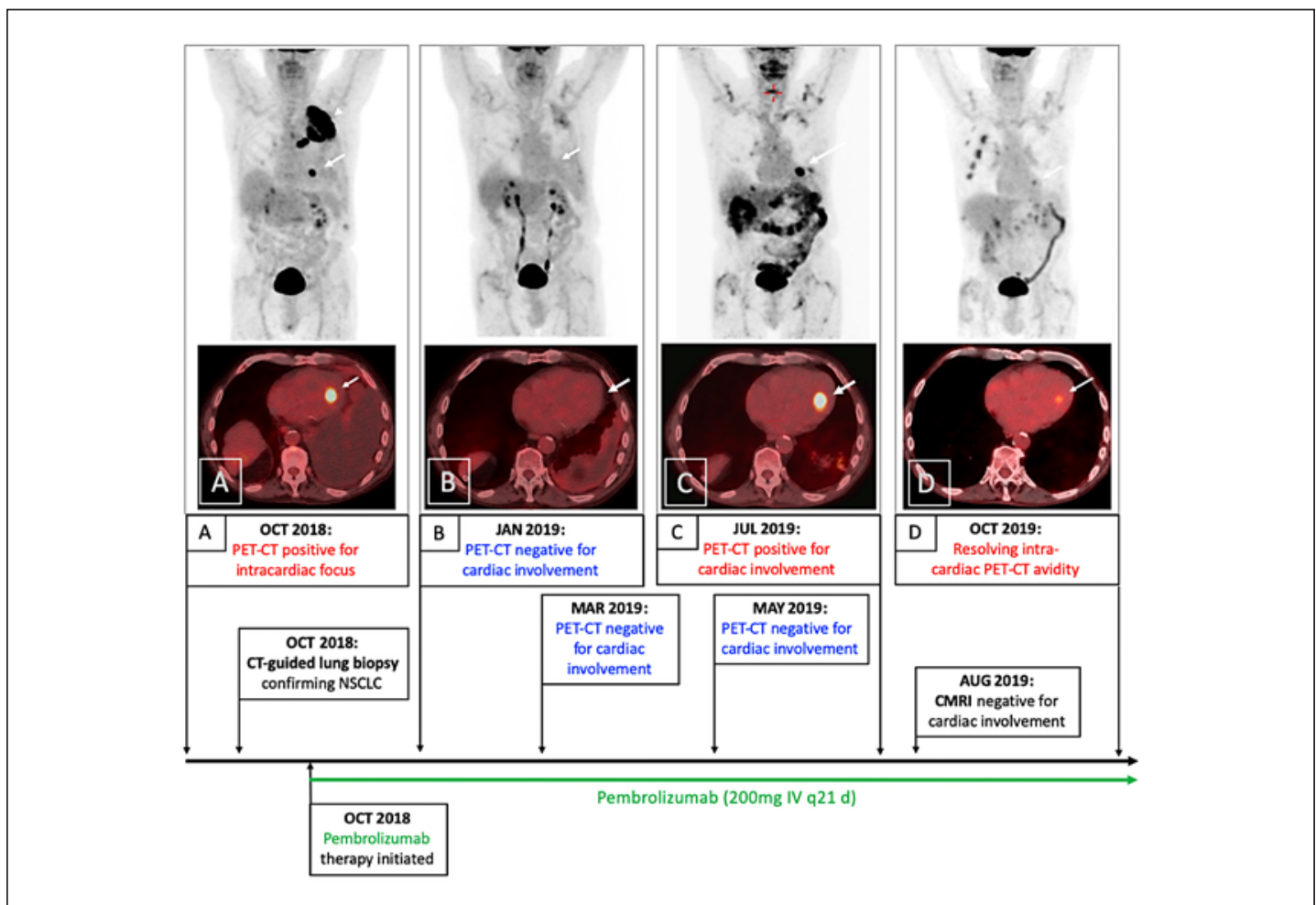

Fig. 1. Timeline of ${ }^{18}$ F-FDG PET-CT findings, tracked with a pembrolizumab regimen and other diagnostic testing modalities. A transient intracardiac ${ }^{18} \mathrm{~F}$-FDG avidity focal point on PET-CT is indicated by white arrows.

\section{Discussion}

The high clinical suspicion of cardiac metastasis in this case was supported by a number of elements surrounding the ${ }^{18} \mathrm{~F}$-FDG PET-CT findings. Most importantly, one must note the timing and the progression of these findings. First identified at a pretreatment stage, the intracardiac uptake seemingly resolved upon initiation of pembrolizumab. This matched the improvement that was also seen at the primary tumor and other sites of metastasis, until eventually the ventricular avidity recurred independently 9 months into treatment. Secondly, diagnostic power was limited by the tests the patient had consented to before he eventually agreed to undergo CMRI. The cardiologists could not rule out the presence of an endomyocardial mass on echocardiography. The EKG findings were unremarkable. The risk of biopsy or surgical resection was considered too high in this 84-year-old male with multiple comorbidities. Alternatively, the recurrence of cardiac avidity was not associated with clinical signs of other potential entities on the differential diagnosis, such as an infectious process like endocarditis (fever, new-onset or worsening murmur, dermatologic or nail bed findings, etc.).

Also notable is the known entity of pseudoprogression. Recurrence of, or an increase in, avidity on ${ }^{18} \mathrm{~F}$-FDG PET-CT most likely represents real disease progression in cases of true metastasis. However, there are reported cases in NSCLC whereby tumor response was preceded by apparent progression on imaging [19-23]. Pseudoprogression is likely due to inflammatory infiltration associated with immunotherapeutic agents [24], and it is usually noted during early stages of treatment. Suyanto et al. [23], however, reported pseudoprogression occurring as late as 15 cycles into treatment with nivolumab. Eshghi et al. [25] 
Bilani et al.: Multimodal Imaging Approach to Mitigate False Positivity in Cardiac Metastasis

described a case of NSCLC that progressed to axillary and supraclavicular lymph nodes 15 weeks into a course of nivolumab. This progression responded favorably 5 weeks later while on the same treatment regimen. This variability in documented pseudoprogression potentially reflects the dynamic tug-of-war between an enhanced immune response and tumor virulence. This process is not limited to the primary site of disease. Kim et al. [22] reported a case of NSCLC with pseudoprogression at a metastatic site causing intestinal perforation.

CMRI performed 1 month later - which was not suggestive of cardiac involvement, at least at the time of imaging - directed the course of management in this case. While echocardiography showed stable cardiac function, findings on PET-CT correlated well to the patient's clinical presentation (i.e., worsened dyspnea, exercise intolerance, etc.). Biopsy or surgical evaluation would have posed a significant risk for this elderly male with multiple comorbidities. Radiation oncology and interventional radiology would have been consulted to explore options for local therapy. Additionally, platinum-based chemotherapy would have been considered to supplement the patient's immune checkpoint inhibitor therapy based on data showing prolonged survival in metastatic NSCLC when pembrolizumab was combined with pemetrexed and a platinum agent [26]. Thus, CMRI spared this patient from additional diagnostic or therapeutic procedures that each carry their own set of risks. Data on the sensitivity and specificity of CMRI compared to other imaging modalities in diagnosing cardiac metastasis are limited. In one cohort study of 59 patients, however, CMRI could differentiate between neoplastic and nonmalignant masses $100 \%$ of the time [9]. As other imaging modalities, it allows for anatomic evaluation. However, the resolution of CMRI imaging also provides more insight into tissue composition. PET-CT is known to be less specific as inflammatory cells will also cause increased FDG avidity, most commonly in the context of infection. However, increased uptake has also been reported in postischemic or infarcted tissue [2729], atherosclerosis [30], physiological papillary muscle [31], hypertrophied papillary muscle [32], and postoperative contexts [32]. To our knowledge, there are no reported cases of chronic mitral regurgitation due to prolapse being a cause of increased FDG avidity. An irregular pattern of ventricular uptake in normal physiological states is not uncommon. Even diet has been shown to alter myocardial uptake, which is why guidelines recommend a lowcarbohydrate diet and prolonged fasting leading up to oncological PET-CT scans [7,33]. Focal areas of increased and decreased uptake should not be considered sites of disease in the absence of a supporting cardiac history, since these may represent normal inter-patient variation [31,34]. Of course, this variation is less clear-cut in real-world cases such as the one presented, where common and dynamic pulmonary or cardiac comorbidities complicate the diagnostic pathway.

The diagnostic and prognostic utility of CMRI continues to evolve, making it the reference standard for evaluation of many cardiac pathologies. As of yet, there are no diagnostic guidelines describing the use of CMRI to rule out cardiac involvement in malignancy. Zhu et al. [9] propose a diagnostic algorithm including CMRI based on one single-center cohort study. This case highlights the significance of a multimodal approach that includes CMRI early in the evaluation in order to help minimize medical resource utilization and radiation/surgical exposure in patients.

\section{Statement of Ethics}

IRB approval was not required for this case report. All information presented is de-identified and the patient has provided consent for this case to be written. 
Bilani et al:: Multimodal Imaging Approach to Mitigate False Positivity in Cardiac Metastasis

\section{Disclosure Statement}

The authors have no conflicts of interest to declare.

\section{Funding Sources}

There were no funding sources associated with this work.

\section{Author Contributions}

N.B.: conceptualization, design, and writing; L.E., F.M., D.S., Z.N.: design, writing, and editing; E.E.: editing; E.A.: project supervision, design, writing, and editing.

\section{References}

1 Choi YH, Han HS, Lim SN, Lee SY, Koo JH, Lee OJ, et al. Multiple cardiac metastases from a nonfunctioning pancreatic neuroendocrine tumor. Cancer Res Treat. 2013;45(2):150-4.

2 Goldberg AD, Blankstein R, Padera RF. Tumors metastatic to the heart. Circulation. 2013;128(16):1790-4.

3 Mukai K, Shinkai T, Tominaga K, Shimosato Y. The incidence of secondary tumors of the heart and pericardium: a 10-year study. Jpn J Clin Oncol. 1988;18(3):195-201.

4 Kim JH, Jung JY, Park Y, Hwang SI, Jung CS, Lee SH, et al. Non-small cell lung cancer initially presenting with intracardiac metastasis. Korean J Intern Med. 2005;20(1):86-9.

5 Sato R, Ichiyasu H, Saeki S, Sakata S, Sasaki J, Fujii K, et al. FDG-PET and chemotherapy for successful diagnosis and treatment of cardiac metastasis from non-small cell lung cancer. Intern Med. 2012;51(14):1909-12.

6 Tamura A, Matsubara O, Yoshimura N, Kasuga T, Akagawa S, Aoki N. Cardiac metastasis of lung cancer. A study of metastatic pathways and clinical manifestations. Cancer. 1992;70(2):437-42.

7 Saponara M, Ambrosini V, Nannini M, Gatto L, Astolfi A, Urbini M, et al. 18F-FDG-PET/CT imaging in cardiac tumors:illustrativeclinical casesand reviewoftheliterature.TherAdvMed Oncol.2018;10:1758835918793569.

8 Dos Santos VM, Dos Santos LAM. Cardiac and lymphatic metastases from lung cancer. Arch Iran Med. 2018;21(2):82-3.

9 Zhu D, Yin S, Cheng W, Luo Y, Yang D, Lin K, et al. Cardiac MRI-based multi-modality imaging in clinical decisionmaking: preliminary assessment of a management algorithm for patients with suspected cardiac mass. Int J Cardiol. 2016;203:474-81.

10 Deng S, Zhang B, Li J, Sang S, Zhang W. Identification of multiple cardiac metastases from nonsmall-cell lung cancer by 18F-FDG PET/CT: a case report. Medicine (Baltimore). 2018;97(49):e12868.

11 Stokes MB, Nerlekar N, Moir S, Teo KS. The evolving role of cardiac magnetic resonance imaging in the assessment of cardiovascular disease. Aust Fam Physician. 2016;45(10):761-4.

12 Hori Y, Funabashi N, Miyauchi H, Nakagawa K, Shimura H, Miyazaki M, et al. Angiosarcoma in the right atria demonstrated by fusion images of multislice computed tomography and positron emission tomography using F-18 fluoro-deoxyglucose. Int J Cardiol. 2007;123(1):e15-e17.

13 Moulin-Romsee G, De Wever W, Verbeken E, Mortelmans L. Atrial metastasis of esophageal carcinoma detected by follow-up FDG PET/CT. Clin Nucl Med. 2007;32(5):393-5.

14 Orcurto MV, Delaloye AB, Letovanec I, Martins Favre M, Prior JO. Detection of an asymptomatic right-ventricle cardiac metastasis from a small-cell lung cancer by F-18-FDG PET/CT. J Thorac Oncol. 2009;4(1):127-30.

15 Coccia P, Ruggiero A, Rufini V, Maurizi P, Attinà G, Marano R, et al. Cardiac metastases of Ewing sarcoma detected by 18F-FDG PET/CT. J Pediatr Hematol Oncol. 2012;34(3):236-8.

16 Derlin T, Clauditz TS, Habermann CR. Adrenal epithelioid angiosarcoma metastatic to the epicardium: diagnosis by 18F-FDG PET/CT. Clin Nucl Med. 2012;37(9):914-5.

17 Vatankulu B, Dirlik Serim B, Sonmezoglu K, Vatankulu MA. Right ventricle metastasis of pleomorfic undifferentiated sarcoma detected by FDG PET/CT and three-dimensional echocardiography. Echocardiography. 2016;33(7):1103-4.]

18 Crombé A, Lintingre PF, Le Loarer F, Lachatre D, Dallaudière B. Multiple skeletal muscle metastases revealing a cardiac intimal sarcoma. Skeletal Radiol. 2018;47(1):125-30.

19 Tanizaki J, Hayashi H, Kimura M, Tanaka K, Takeda M, Shimizu S, et al. Report of two cases of pseudoprogression in patients with non-small cell lung cancer treated with nivolumab - including histological analysis of one case after tumor regression. Lung Cancer. 2016;102:44-8. 
20 Izumida T, Kawagishi Y, Tsuji H. Pseudoprogression in lung adenocarcinoma during treatment with nivolumab. BMJ Case Rep. 2017;2017:bcr-2017-219919.

21 Liu G, Chen T, Li R, Zhu L, Liu D, Ding Z. Well-controlled pleural effusion indicated pseudoprogression after immunotherapy in lung cancer: a case report. Thorac Cancer. 2018;9(9):1190-3.

22 Kim HK, Baek SW, Jeong Y, Yang Y, Kwon J, Han HS, et al. Pseudoprogression presenting as intestinal perforation in non-small cell lung cancer treated with anti-PD-1: a case report. Mol Clin Oncol. 2019;11(2):132-4.

23 Suyanto S, Yeo D, Khan S. A rare delayed atypical pseudoprogression in nivolumab-treated non-small-cell lung cancer. Case Rep Oncol Med. 2019;2019:8356148.

24 Higuchi M, Suzuki H, Gotoh M. Role of PET/computed tomography in radiofrequency ablation for malignant pulmonary tumors. PET Clin. 2016;11(1):47-55.

25 Eshghi N, Lundeen TF, Kuo PH. Dynamic adaptation of tumor immune response with nivolumab demonstrated by 18F-FDG PET/CT. Clin Nucl Med. 2018;43(2):114-6.

26 Gandhi L, Rodríguez-Abreu D, Gadgeel S, Esteban E, Felip E, De Angelis F, et al. Pembrolizumab plus chemotherapy in metastatic non-small-cell lung cancer. N Engl J Med. 2018;378(22):2078-92.

27 Dilsizian V. 18F-FDG uptake as a surrogate marker for antecedent ischemia. J Nucl Med. 2008;49(12): 1909-11.

28 Wollenweber T, Roentgen P, Schäfer A, Schatka I, Zwadlo C, Brunkhorst T, et al. Characterizing the inflammatory tissue response to acute myocardial infarction by clinical multimodality noninvasive imaging. Circ Cardiovasc Imaging. 2014;7(5):811-8.

29 Rischpler C, Dirschinger RJ, Nekolla SG, Kossmann H, Nicolosi S, Hanus F, et al. Prospective evaluation of 18F-FDG uptake in post-ischemic myocardium by simultaneous PET/MRI as a prognostic marker of functional outcome. Circ Cardiovasc Imaging. 2016;9(4):e004316.

30 Carter KR, Kotlyarov E. Common causes of false positive F18 FDG PET/CT scans in oncology. Braz Arch Biol Technol. 2007;50(SPE):29-35.

31 Özülker T, Özülker F. Normal variants and benign findings. In: Özülker T, Özülker F, editors. Atlas of PET-CT imaging in oncology. Springer;2015. pp. 21-158.

32 Fathala A, Abouzied M, AlSugair AA. Cardiac and pericardial tumors: a potential application of positron emission tomography-magnetic resonance imaging. World J Cardiol. 2017;9(7):600-8.

33 Bogsrud TV, Lowe VJ. Normal variants and pitfalls in whole-body PET imaging with 18F FDG. Appl Radiol. 2006;35(6):16-30.

34 de Groot M, Meeuwis AP, Kok PJ, Corstens FH, Oyen WJ. Influence of blood glucose level, age and fasting period on non-pathological FDG uptake in heart and gut. Eur J Nucl Med Mol Imaging. 2005;32(1):98-101. 